

Cite this: J. Mater. Chem. B, 2014, 2 , 6144

Received 9th April 2014

Accepted 7th July 2014

DOI: $10.1039 / c 4 t b 00563 e$

www.rsc.org/MaterialsB

\section{Carbon nanomaterials as new tools for immunotherapeutic applications}

\begin{abstract}
Alessia Battigelli, Cécilia Ménard-Moyon and Alberto Bianco*
Carbon-based nanomaterials, including carbon nanotubes and graphene, have gained great attention in the scientific community due to their unique physico-chemical properties, which could be also promising in many biomedical-related fields. In particular, their low cytotoxicity, achieved when properly functionalized, along with the possibility to link multiple bioactive molecules, realistically allows envisaging their potential use as a therapeutic platform. In this context, the immune system and immune responses play an important role in our organism, as they are involved either directly or indirectly in many diseases. Therefore, the possibility to prevent or block a disease by controlling and/or modulating the immune responses has become an important task in nanomedicine. In this feature article the advantages of using carbon-based materials in immunotherapy are presented. Important goals achieved using carbon nanotubes and graphene are described, highlighting the promising use of these nanomaterials in cancer treatment, imaging and vaccine development. The capacity of functionalized carbon nanotubes to modulate the immune responses is also discussed, highlighting the current state of the art and the future developments on this subject.
\end{abstract}

\section{Introduction}

In recent years, nanotechnology has been gaining great interest in the field of medicine, due to the unique characteristics of nanomaterials, promising tools as drug delivery systems or

CNRS, Institut de Biologie Moléculaire et Cellulaire, Laboratoire d'Immunopathologie et Chimie Thérapeutique,67000 Strasbourg, France. E-mail: a.bianco@ibmc-cnrs. unistra.fr contrast agents for imaging. ${ }^{1,2}$ Immunotherapy is one important branch of medicine dealing with the induction, the enhancement, or the suppression of an immune response. Our immune system is designed to recognize and neutralize foreign antigens or exogenous molecules. The identification of antigens is assured by the antibodies that recognize specific receptors present on the surface of the cells. The specific antigen-antibody affinity is widely exploited in immunotherapy for the recognition of particular types of cells. One of the first

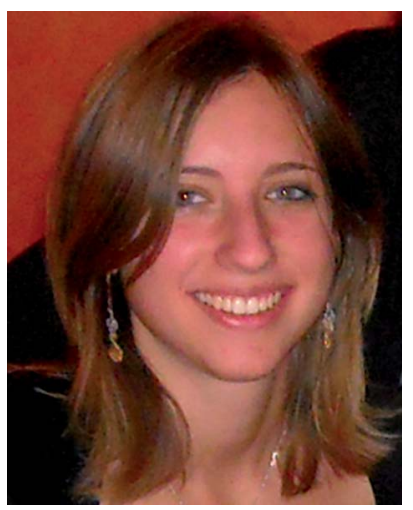

Alessia Battigelli obtained her "Laurea" degree from the University of Trieste in 2008. After her graduation, she was awarded the Vinci Fellowship and started a joined PhD program in the groups of Prof. Maurizio Prato (University of Trieste) and Dr Alberto Bianco (CNRS, Strasbourg), working on the chemical functionalization of carbon nanotubes for drug delivery purposes. She is currently a postdoctoral fellow in the group of Dr Ron Zuckermann at the Lawrence Berkeley National Laboratory in the USA. Her research interests include the functionalization of nanomaterials and their biological applications in the field of drug delivery, biosensing and molecular recognition.

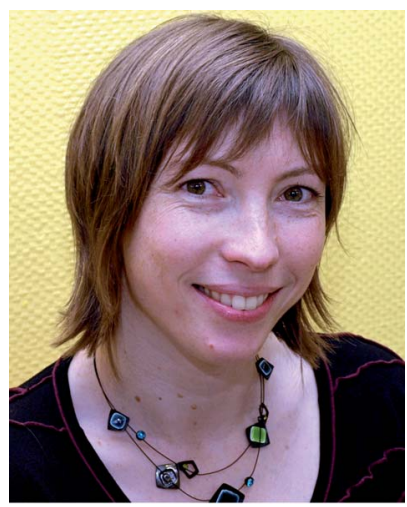

Dr Cécilia Ménard-Moyon obtained her PhD in 2005 at CEA/Saclay in France on carbon nanotubes, their applications for optical limitation, nanoelectronics, and on the development of novel methods of functionalization. After 1 year post-doc at the University of York (U. K.) on the total synthesis of a natural product, she joined the R\&D Department of Nanocyl in Belgium for 18 months to work on the synthesis and functionalization of carbon nanotubes. Since 2008, she has been a researcher at the CNRS in the group of A. Bianco in Strasbourg. Her research interests focus on the functionalization of carbon-based nanomaterials for therapeutic and imaging applications. 



Fig. 1 Transmission electron microscopy (TEM) images of MWCNTs (A) and graphene oxide (B).

applications of antibodies in medicine was associated with the development of new vaccines, which are engineered to mimic antigens and induce an immune response in a host to prevent diseases, including infectious diseases. In the case of cancer, particular proteins are overexpressed on the surface of cancer cells and the use of specific antibodies to target them allows imaging of cancer cells and opens possibilities for anticancer treatment.

Carbon nanotubes (CNTs) have been described at the atomic level for the first time in 1991 by Iijima. ${ }^{3}$ They can be classified as single-walled CNTs (SWCNTs) and multi-walled CNTs (MWCNTs), depending on the number of graphene shells coaxially arranged to form the tubes (Fig. 1A). Since their discovery, CNTs have been intensively investigated due to their particular physico-chemical properties in many scientific fields, such as electronics, sensing, composite materials science, and nanobiotechnology. ${ }^{4}$ In particular, their ability to cross biological barriers promoted their study in nanomedicine. ${ }^{5}$ The isolation of graphene is more recent. ${ }^{6}$ Graphene is a two dimensional material formed by a single layer of $\mathrm{sp}^{2}$-bonded carbon atoms with outstanding electronic, mechanical, and optical properties associated with a high specific surface area, thus making it a promising tool in nanotechnology. A derived graphene nanomaterial is graphene oxide (GO). GO is currently

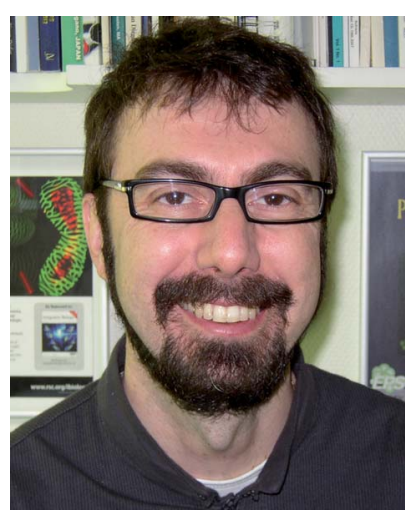

Dr Alberto Bianco received his $\mathrm{PhD}$ in 1996 from the University of Padova (Italy). As a visiting scientist, he worked at the University of Tübingen as an Alexander von Humboldt fellow (1996-1997) and at the University of Padova (1997-1998). He is currently a Research Director at the CNRS in Strasbourg (France). His research interests focus on the design and functionalisation of carbon-based nanomaterials and their use for therapeutic, diagnostic and imaging applications. He is the author of over 180 papers. He is in the Advisory Board of Nanomedicine, Nanotechnology Reviews and the Journal of Peptide Science, and the Editor of Carbon. widely exploited in particular in the field of nanomedicine, as a biosensor or a drug delivery system. The presence of oxygenated functional groups such as hydroxyl, epoxy, carbonyl, and carboxyl groups enhances the dispersibility of GO in water and offers the possibility to further functionalize its surface (Fig. 1B). ${ }^{7}$

The intrinsic capability of nanomaterials to accumulate in the tumor, due to the enhanced permeability and retention (EPR) effect, renders them appealing candidates in the treatment of cancer by immunotherapy. ${ }^{8}$ In this context, carbonbased nanomaterials (e.g. carbon nanotubes and graphene) have recently attracted great interest in the field of nanomedicine ${ }^{9-12}$ and due to their physico-chemical features they are considered emerging candidates in the field of immunotherapy. One important characteristic is the possibility to multifunctionalize their surface to impart multiple functionalities via covalent or non-covalent modification ${ }^{13-15}$ or a combination of the two. ${ }^{16}$ For example, it is possible to increase the solubility of the material using a hydrophilic chain like triethylene glycol (TEG) or polyethylene glycol (PEG). ${ }^{17-19}$ They can also be conjugated to a drug and/or a specific targeting moiety. ${ }^{20}$ Moreover, functionalization allows minimizing cytotoxicity ${ }^{21}$ and immunogenicity. ${ }^{22,23}$ In this direction we recently studied the in vivo impact of different types of functionalization. ${ }^{21} \mathrm{High}$ levels of inflammatory reaction were observed in $\mathrm{C} 57 \mathrm{Bl} / 6$ mice when long unmodified or alkylated CNTs were intraperitoneally injected. In contrast, short CNTs functionalized with an aminoterminal TEG chain induced no inflammation, revealing the importance of CNT length and surface decoration as crucial parameters to consider when designing novel carbon nanotubebased therapeutic tools. Additionally, the degree of CNT functionalization was also analyzed as it can influence the biodistribution and excretion profile. ${ }^{24}$

In this feature article, we present the most relevant studies describing the use of carbon-based materials in immunotherapy and their effects on the immune system. Initially, the possible application in nanomedicine will be discussed highlighting some examples in cancer treatment, imaging and vaccination in which carbon nanomaterials were successfully used exploiting their interaction with the immune system after appropriate functionalization. Then, we will describe the effect of carbon-based nanomaterials on the immune system.

\section{Cancer immunotherapy}

The development of new drug delivery systems for the treatment of cancer is one of the most investigated challenges in nanomedicine nowadays. The possibility to use antibodies to specifically recognize and target tumor cells is an interesting approach and different nanocarriers have been studied so far. ${ }^{1}$ The use of carbon nanotubes to carry antibodies gives some important advantages. The combination of the targeting properties of antibodies with the intrinsic capability of CNTs to absorb near infra-red (NIR) light for photothermal therapy, and the possibility to multifunctionalize CNTs with different therapeutic cargos are only some examples (Table 1). 
Table 1 Characteristics of CNT and graphene conjugates used for cancer immunotherapy

\begin{tabular}{|c|c|c|c|c|c|}
\hline $\begin{array}{l}\text { Type of carbon } \\
\text { nanomaterial }\end{array}$ & Bioactive molecules & Functionalization & In vitro studies & In vivo studies & Reference \\
\hline SWCNTs & Anti-HER2 and anti-IGF1R & Non-covalent & MCF-7 cells & - & 25 \\
\hline SWCNTs & Anti-HER2 & Covalent & SK-BR-3 cells & - & 26 \\
\hline SWCNTs & RFB4 and RFT5 & Non-covalent / covalent & Daudi cells and PBMCs & - & 27 and 28 \\
\hline MWCNTs & $\mathrm{H} 22 \mathrm{p}$ & Covalent & - & $\mathrm{BALB} / \mathrm{c}$ mice & 29 \\
\hline SWCNTs & $\mathrm{CpG}$ & Non-covalent & BMM and GL261 cells & C57BL/6 mice & 31 and 33 \\
\hline GO and sGO & $\mathrm{CpG}$ & Covalent & RAW264.7 cells & - & 34 \\
\hline SWCNTs & SOCS1-SiRNA & Non-covalent & Bone marrow-derived DC & C57BL/6 mice & 35 \\
\hline SWCNTs & anti-P-gp/doxorubicin & Non-covalent / covalent & K562S and K562R cells & - & 37 \\
\hline SWCNTs & anti-GITR & Non-covalent & - & $\mathrm{C} 57 \mathrm{BL} / 6 \mathrm{~J}$ mice & 38 \\
\hline
\end{tabular}

Different studies demonstrated the enhanced effect in the conjugation of targeting antibodies to CNTs, followed by photothermal treatment by NIR irradiation. One of the first examples was reported in 2007 by the group of Panchapakesan. ${ }^{25}$ In this study, 1-pyrenebutanoyl succinimide was non-covalently attached by $\pi-\pi$ interactions on the surface of SWCNTs. After grafting a PEG chain to increase the dispersibility of the material, the antibodies were linked to pyrene via the reaction with a succinimide moiety (Fig. 2A). Two mouse monoclonal antibodies (anti-HER2 and anti-IGF1R) were chosen for their ability to specifically recognize human endothelial receptor 2 (HER2) and insulin-like growth factor 1 receptor (IGF1R), respectively. These two receptors are overexpressed in a broad number of cancer cells and they can be considered potential markers for tumors. SWCNTs functionalized with fluorescently labeled antiHER2 or anti-IGF1R were incubated into MCF-7 breast cancer cells. After 30 minutes, a high cellular uptake of the conjugates was observed confirming the effective targeting mediated by the antibodies. MCF-7 cells, that internalized both anti-HER2-CNTs and anti-IGF1R-CNTs, were irradiated using NIR light (808 nm) to induce cell death. The thermal ablation effect was particularly effective on the cells targeted by the nanotubes functionalized with the combination of the two antibodies.
Similar results were achieved by Xiao et al.,${ }^{26}$ but in this case the authors attached covalently the anti-HER2 antibody to oxidized SWCNTs after activation of the carboxylic groups (Fig. 2B). SK-BR-3 breast carcinoma cells, over-expressing HER2 receptors, were incubated with the antibody-CNTs and, different from the previous study, CNTs were found localized only on the cellular membrane and not internalized inside the cells. The subsequent NIR irradiation at $808 \mathrm{~nm}$ caused extensive cell death only in the cells treated with the antibody-CNTs and not in the cells incubated with CNTs lacking the antibody or without CNTs.

By exploiting a synthetic approach similar to that described by Panchapakesan and coworkers, ${ }^{25}$ the group of Vitetta reported the non-covalent functionalization of SWCNTs with DSPEPEG(2000)-biotin (1,2-distearoyl-sn-glycero-3-phosphoethanolamine- $N$-[biotinyl(polyethyleneglycol)2000]). ${ }^{27}$ Before attaching the antibody to biotinylated-CNTs, neutralite avidin was firstly activated and coupled to the two thiolated specific IgGs: RFB4, a mouse IgG anti-human CD22, and RFT5, a mouse IgG antihuman CD25. The strong affinity between biotin and avidin allowed linking the antibodies to CNTs (Fig. 2C). The conjugates were then incubated with cells expressing the specific receptors, CD22 or CD25 (Daudi cells and peripheral blood mononuclear

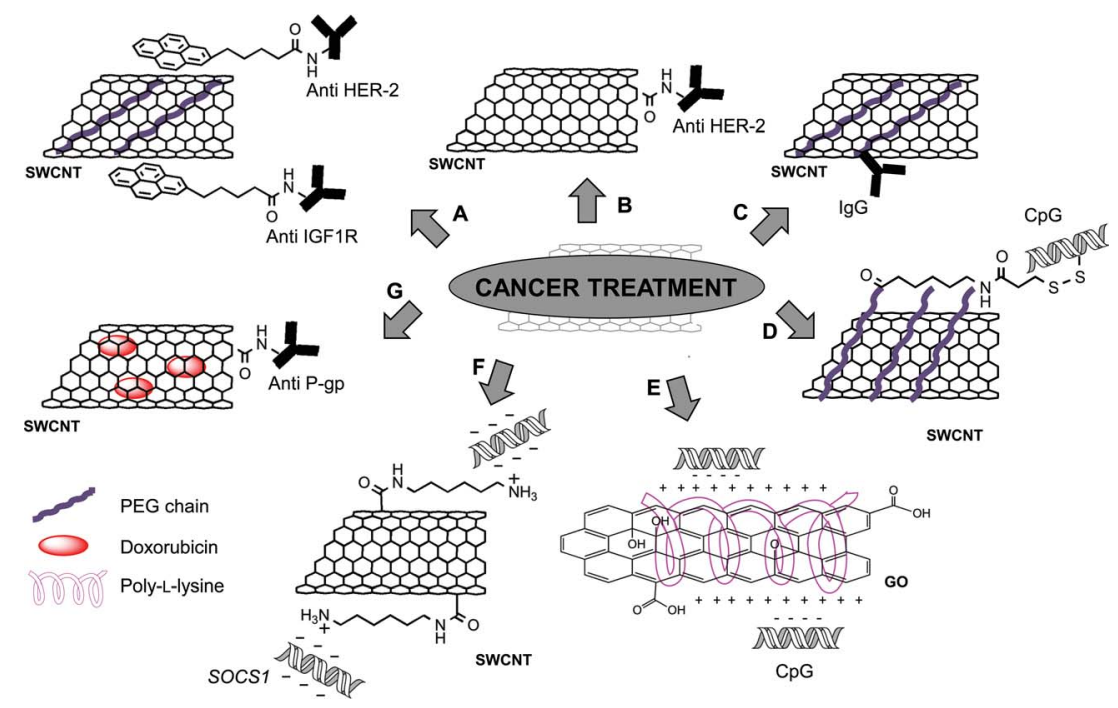

Fig. 2 Different strategies using functionalized CNTs and graphene for the treatment of cancer. 
cells, PBMCs, respectively). Targeted cells were then exposed to NIR irradiation at $808 \mathrm{~nm}$ to trigger cell death by thermal ablation. Similar results were reported later by the same group ${ }^{28}$ investigating a covalent approach to functionalize oxidized SWCNTs with RFB4 and RFT5 IgGs. These results highlight the efficacy of using CNTs conjugated with antibodies to specifically target cancer cells and the relevance of this approach in nanomedicine.

In 2008, the group of Yang reported the potential of CNTs for the immunotherapeutic treatment of cancer in vivo. ${ }^{29}$ The purpose of this work was to study the possibility to enhance the anticancer immune reaction of tumor cell vaccines (TCVs) by using CNTs as delivery systems. For this purpose, oxidized MWCNTs were covalently conjugated to tumor lysate proteins (H22p), a mixture of various tumor proteins derived from mouse H22 liver cancer cells. One week after inoculation of H22 cancer cells, BALB/c mice were treated with TCVs and H22pCNTs. After 90 days, about $55 \%$ of the mice were cured from cancer when treated with H22p-CNTs. This value was significantly higher than the control mice group treated with only TCV or a mixture of TCVs and H22p $37.5 \%$ of cured mice in both cases) and the control with TCVs and CNT lacking H22p (45.6\% of cured mice). Splenic lymphocytes of mice treated with TCVs and H22p-CNTs showed an enhanced antitumor cytotoxic effect. Histological studies of the tumor tissues showed high lymphocyte infiltration with tumor necrosis, whereas no lymphocytes were observed in the tumor of the controls, suggesting the absence of an antitumor immune reaction. To show the specificity of the antitumor immunity, two types of cancer cells, H22 and then two weeks later epithelial-mesenchymal transition cells, were injected into the same mice, cured after the first treatment. Mice were able to reject the inoculated $\mathrm{H} 22$ cancer cells, but in the second case the tumor grew forming a consistent mass. Even if the exact immune mechanism was difficult to explain by the authors, this work demonstrates the specific antitumor immune response enhanced by CNTs conjugated with $\mathrm{H} 22 \mathrm{p}$. The most reasonable mechanism hypothesized by the authors relies on the capability of CNTs as delivery systems, which could carry efficiently $\mathrm{H} 22 \mathrm{p}$ to the antigen presenting cells of the immune system.

Another antitumor immuno-strategy recently developed involves the functionalization of CNTs with a stimulator of both innate and adaptive immune systems, namely CpG oligodeoxynucleotides. ${ }^{30,31}$ CpGs are known ligands for intracellular toll-like receptor-9 (TLR9), which after CpG stimulation leads to the inhibition of tumor growth as demonstrated in a glioma model. ${ }^{32}$ For this purpose, SWCNTs were used to enhance the cellular uptake of CpGs, which alone showed a low capacity to cross the cell membrane and low physiological stability. In our group, we investigated the effect of positively charged CNTs complexed with ODN CpG 1668 on the immune system in vitro, observing an increased immunostimulation. ${ }^{30}$ More recently, the group of Badie reported a different functionalization of SWCNTs with CpGs, in which CNTs were firstly modified non-covalently with a DSPE-PEG chain. ${ }^{31}$ The PEG chain was then further modified with a sulfhydryl linker, for the conjugation of a thiolated CpG through a cleavable disulfide bond (Fig. 2D). The in vitro studies using bone marrow-derived monocytes (BMM) and GL261 glioma cells incubated with CNTs revealed that the conjugation of CpGs to CNTs not only enhanced the uptake of the oligodeoxynucleotides, but also the expression of proinflammatory cytokines and chemokines, due to their immunostimulator properties. Importantly, similar results were obtained in vivo after injection of CNTs into mice implanted with glioma cells. In addition, a slower clearance of CpGs was observed, in comparison with free CpGs. The combination of these effects not only resulted in a delayed tumor growth, but also about $60 \%$ of the treated mice were cured. GL261 glioma cells were injected again into these mice, and their survival demonstrated that the mice developed a full immune protection against this type of cancer. A similar strategy was then investigated for the treatment of metastatic brain tumor. ${ }^{33}$ For this experiment B16 melanoma cells were inoculated into $\mathrm{C} 57 \mathrm{BL} / 6$ mice both intracranially (i.c.) and subcutaneously (s.c.). Interestingly, it was observed that CNTs administered i.c. abrogated the growth of both i.c. and s.c. melanomas with a higher inflammatory response in comparison with the CNTs injected s.c., which instead caused only a predominant local response, with few benefits on the i.c. tumor. These findings suggest that intracranially injected CpG-CNTs have potential not only in the treatment of glioma but also to cure metastatic brain tumor.

Very recently, the same approach was explored by Sun et al. by using GO as a nanocarrier. ${ }^{34} \mathrm{GO}$ and small graphene oxide (sGO) were both covalently functionalized with poly-L-lysine. Negatively charged $\mathrm{CpG}$ oligonucleotides were immobilized onto functionalized GO via electrostatic interactions with the amino groups of poly-L-lysine (Fig. 2E). Using a labeled CpGCy3, the cellular internalization of the GO-based conjugates into RAW264.7 macrophages was initially assessed. The immunostimulatory activity of the conjugates was also studied by measuring the level of secreted cytokines. The immune response observed by the secretion of tumor necrosis factor $\alpha$ (TNF- $\alpha$ ) and interleukin 6 (IL-6) suggested a prompt delivery of CpG, highlighting in particular the capability of sGO to be an effective immunostimulator. The immunostimulatory activity of the GO-based conjugates has potential use as a therapeutic tool in cancer treatment.

In 2006, the group of Chen prepared complexes of SWCNTs and siRNA (small interfering RNA) to develop efficient in vivo cancer treatment. ${ }^{35}$ CNTs were initially functionalized with 1,6diaminohexane to obtain a positively charged material. Then, as the selected gene to be silenced, the researchers chose the suppressor of cytokine signaling 1 (SOCS1) located into dendritic cells (DCs), one of the most efficient types of antigenpresenting cells (Fig. 2F). Indeed, the silencing of this specific intracellular signal regulator was previously demonstrated to enhance antitumor immunity. ${ }^{36}$ After complexation of SWCNTs with SOCS1-siRNA, the silencing effect was assessed in bone marrow-derived DC antigen-presenting cells. It was also observed that the silencing of SOCS1 caused an increase of the antigen-presenting functions of DCs. In vivo studies were conducted on $\mathrm{C} 57 \mathrm{BL} / 6$ mice, by administration of the SOCS1siRNA/CNT complexes one day after B16 tumor cell inoculation. 
An effective retardation on tumor growth was observed after 15 days, confirming that the animals developed antitumor immunity.

To overcome the problem of multidrug resistance in cancer treatment, Li et al. ${ }^{37}$ functionalized CNTs with doxorubicin and an antibody recognizing the P-glycoprotein (anti-P-gp), which is overexpressed in multidrug resistant cells. This overexpression causes an increased efflux of anticancer drugs. In this work, the antibody was covalently bound to the carboxylic group of oxidized SWCNTs by amidation. Doxorubicin was then adsorbed onto the nanotube surface by $\pi-\pi$ stacking (Fig. $2 \mathrm{G}$ ). The capability of the constructs to target specifically multidrug resistant tumor cells was studied using human leukemia cells sensitive or resistant to the drug (K562S and K562R, respectively), revealing a more than 20-fold higher targeting of multidrug resistant cells than the others. Cells were then exposed to NIR radiation to induce the release of the drug from the CNT surface. A significant enhanced cytotoxic effect was observed in comparison with the free doxorubicin, confirming the promising properties of this drug delivery system.

In a recent study, Sacchetti et al. showed the possibility to target regulatory $\mathrm{T}$ cells $\left(\mathrm{T}_{\mathrm{reg}}\right)$ using SWCNTs functionalized with a specific antibody. ${ }^{38} \mathrm{~T}_{\mathrm{reg}}$ is a subset of $\mathrm{T}$ cells, which is intensively studied for tumor immunotherapy because it is exploited by tumors to protect itself from host antitumor responses. In this work, the sidewalls of SWCNTs were noncovalently functionalized with PEG-modified phospholipids and then a specific targeting antibody was anchored directly on the PEG chain, via a biotin-neutravidin interaction. The selected ligand was DTA-1, a specific antibody for GITR (glucocorticoid-induced TNFR-related receptor), which is a marker preferentially expressed on the surface of $\mathrm{T}_{\text {reg }}$ and is more overexpressed in intratumor cells than in peripheral cells. In vivo experiments were conducted on $\mathrm{C} 57 \mathrm{BL} / 6 \mathrm{~J}$ mice bearing B16 melanoma, using DTA-1-SWCNTs labeled with a NIRemitting fluorophore. The targeting of $\mathrm{T}_{\text {reg }}$ cells was confirmed as the majority of CNTs was found into these cells and not into the other cells. In particular, the CNT conjugate was uptaken preferentially in the tumor compared to other tissues, as a consequence of the EPR effect and of the selective and efficient targeting of tumor $\mathrm{T}_{\text {reg }}$ by DTA- 1 .

Overall, the studies conducted on the use of CNTs conjugated to antibodies for cancer treatment highlight the versatility of this type of nanomaterials. The extended available surface of the nanotubes allows the covalent binding or the adsorption of different bioactive molecules at high density. Moreover, the intrinsic physico-chemical properties of CNTs allow enhancing the cellular uptake of ligands attached to the tubes, thus allowing to modulate the immune responses. In addition, the capability of CNTs to absorb NIR light opens attractive opportunities to kill targeted cancer cells.

\section{Imaging}

The high specific surface area is one of the key characteristics of carbon-based nanomaterials that permits to append multiple molecules on their surface. This is particularly interesting for imaging applications, because this approach allows the anchoring of a targeting antibody and a radioisotope, offering the possibility for in vivo imaging of cancer by different techniques, such as PET (positron emission tomography) or SPECT (single-photon emission computed tomography) (Table 2).

The group of Scheinberg did pioneering studies on the functionalization of CNTs with antibodies and their use in vitro and in vivo. In 2007, they reported the functionalization of SWCNTs with a specific antibody for the targeting of CD20 on human Burkitt lymphoma cells. ${ }^{39}$ SWCNTs were first functionalized by 1,3-dipolar cycloaddition using a TEG chain. Then, a fraction of the terminal amine groups was further modified with DOTA (1,4,7,10-tetraazacyclododecane-1,4,7,10-tetraacetic acid), whereas the remaining free amine functionalities were derivatized with a maleimide linker. This latter reactive group was the site for the conjugation of the targeting anti-CD20 antibody (Rituximab) via sulfhydryl groups introduced by the Traut's reagent (Fig. 3A). After labeling Rituximab-CNTs with a fluorescent probe, the material was incubated with $\mathrm{CD} 20^{+}$ Daudi cells and the targeting of the conjugates was assessed by flow cytometry. The radiolabeled conjugate was then investigated in vivo after chelation of DOTA with ${ }^{111}$ In. $\mathrm{GFP}^{+} / \mathrm{FFLuc}^{+}$ Daudi cells were inoculated into SCID mice and after 28 days the radioactive CNTs with or without the targeting antibody were injected. Biodistribution studies revealed that the effective targeting of the tumor was obtained only with Rituximab-CNTs, whereas no specific tumor localization was observed for the radiolabeled material alone.

In another work using a similar synthesis approach, SWCNTs were functionalized with the E4G10 antibody that targets the monomeric vascular endothelial-cadherin expressed in the tumor angiogenic vessels. ${ }^{40}$ As chelating agents, DOTA and DFO (desferrioxamine B) were used for the complexation of ${ }^{225} \mathrm{Ac}$ or ${ }^{89} \mathrm{Zr}$, respectively (Fig. 3B). In vivo studies conducted on NCr mice bearing xenograft tumors showed that the targeting was effective. By using ${ }^{89} \mathrm{Zr}$-E4G10-SWCNTs, it was possible to observe by PET a rapid and specific accumulation of CNTs into the tumors. The presence of the second radionuclide was exploited for its characteristic decay through emitting cytotoxic alpha particles. Administration of ${ }^{225} \mathrm{Ac}-\mathrm{E} 4 \mathrm{G} 10-\mathrm{SWCNTs}$ into the tumor-bearing mice led to a clear decrease of the tumor volume. This work highlights the versatility of functionalized CNTs. Indeed, by using the same conjugate and changing only the type of radioisotope, the functionalized CNTs can be used in combined modalities for imaging with ${ }^{89} \mathrm{Zr}$, and for therapeutic intervention with ${ }^{225} \mathrm{Ac}$.

Recently, the same group reported an innovative two-step approach to target tumor cells using SWCNTs. ${ }^{41}$ This strategy relies on the administration of a tumor-selective antibody followed by the administration of a cytotoxic drug or an imaging probe with high affinity for the antibody. This permits a first pre-targeting by a long-circulating antibody, which is usually a slow process, and a subsequent second targeting mediated by the interaction between the antibody and a specific recognition molecule. This second step is preferred to be fast to achieve a rapid clearance of the cytotoxic agent. In this context, CNTs were used for their capacity to carry the therapeutic/diagnostic 
Table 2 Characteristics of CNT and graphene conjugates used for imaging

\begin{tabular}{|c|c|c|c|c|c|}
\hline $\begin{array}{l}\text { Type of carbon } \\
\text { nanomaterial }\end{array}$ & Bioactive molecules & Functionalization & In vitro studies & In vivo studies & Reference \\
\hline SWCNTs & Rituximab $/{ }^{111}$ In & Covalent & Daudi cells & SCID mice & 39 \\
\hline SWCNTs & Anti-E4G10 $/ 225 \mathrm{Ac}$ and anti-E4G10/ ${ }^{89} \mathrm{Zr}$ & Covalent & - & NCr mice & 40 \\
\hline SWCNTs & $\mathrm{MORF} /{ }^{111} \mathrm{In}$ and $\mathrm{MORF} /{ }^{225} \mathrm{Ac}$ & Covalent & Daudi cells & SCID mice & 41 \\
\hline GO and rGO & Anti-TRC $105 /{ }^{64} \mathrm{Cu}$ and anti-TRC $105 /{ }^{66} \mathrm{Ga}$ & $\begin{array}{l}\text { Covalent and } \\
\text { non-covalent }\end{array}$ & HUVEC and MCF-7 cells & $\begin{array}{l}\text { Mice bearing } \\
4 \mathrm{~T} 1 \text { tumor }\end{array}$ & $43-45$ \\
\hline GO & Trastuzumab/ ${ }^{111}$ In & Covalent & MDA-MB-231 & BALB mice & 46 \\
\hline
\end{tabular}



Fig. 3 Different functionalizations of CNTs used for imaging.

agent and the recognition molecule. A morpholino-modified 18mer oligonucleotide (MORF) was used as a complementary recognition unit to bind the antibodies to CNTs through selfassembly of oligonucleotide base-pairing. ${ }^{42}$ From a synthetic point of view, the functionalization of SWCNTs was performed by 1,3-dipolar cycloaddition to introduce pyrrolidine rings onto the nanotube surface. A fraction of the terminal amine functions was modified with the morpholino oligonucleotides. The remaining amine groups were derivatized with DOTA for chelation of ${ }^{111}$ In or ${ }^{225}$ Ac (Fig. 3C). Preliminary in vitro studies showed the effective tumor targeting and the recognition between the antibodies linked to their cell receptor and the CNTs, which form clusters on the cellular membrane. SCID mice with xenografted solid tumor were first pre-treated with specific MORF-antibodies and then MORF- ${ }^{111}$ In-SWCNTs were administered into mice. The accumulation of the CNTs into the different organs was quantified, revealing a specific retaining into the tumor, due to the interactions between the antibody 
and the MORF moiety on the CNTs. Following these promising results, MORF- ${ }^{225}$ Ac-SWCNTs were then tested for anticancer activity. Mice implanted with Daudi lymphoma cells $\left(\mathrm{CD} 20^{+} \mathrm{B}\right.$ cells) were treated with specific MORF-antiCD20 antibodies and subsequently with MORF- ${ }^{225}$ Ac-SWCNTs. This multistep approach resulted in an effective eradication of the tumor 15 days after the treatment, revealing the therapeutic potential of this novel targeting strategy.

Graphene and in particular GO are considered as promising alternative materials to CNTs and since their discovery many groups have shown their potential applications in nanomedicine. ${ }^{11}$ As GO contains different oxygenated species, it is dispersible in water and it presents attractive properties for biological applications.

The group of Cai demonstrated the effective possibility to use antibody functionalized- and radiolabeled-graphene for targeting tumor with the final purpose to use them for PET imaging. In different studies, reduced graphene oxide (rGO) ${ }^{43}$ (Fig. 4A) or GO ${ }^{44,45}$ (Fig. 4B) was functionalized with a PEG chain non-covalently or covalently, respectively, and then the TRC105 antibody was covalently coupled to the polymer. This antibody is a murine chimeric IgG1 monoclonal antibody, which specifically binds to both human and murine CD105 (endoglin), almost exclusively expressed on proliferating tumor endothelial cells, thus resulting as an ideal marker for tumor angiogenesis. Amino-PEG modified GO or rGO was partly labeled with FITC (fluorescein isothiocyanate) or NOTA (1,4,7-triazacyclononane1,4,7-triacetic acid), a chelator for $\mathrm{Ga}$ and $\mathrm{Cu}$. The remaining free amines of PEG were then linked to TRC105 antibody via a maleimido moiety. ${ }^{64} \mathrm{Cu}$ (on GO or on rGO) ${ }^{43,44}$ or ${ }^{66} \mathrm{Ga}$ (on GO) ${ }^{45}$ was complexed to NOTA present on the PEG chain. HUVEC or MCF-7 cell lines, which are characterized by a high and low expression of the CD105 receptor, respectively, were incubated with the conjugates. The high fluorescence signals detected into HUVEC and not into MCF-7 cells were indicative of efficient targeting. After complexation with radioactive ${ }^{66} \mathrm{Ga}$ or ${ }^{64} \mathrm{Cu}$ isotopes, the conjugates were administered into mice bearing 4T1 tumor. PET analysis revealed a significant level of the graphene material in the tumor area, confirmed by histological analysis of the tissues. Immunofluorescence analysis was then performed ex vivo on slices of specific tissues, staining CD105 with TRC105-graphene and the vasculature using CD31. A colocalization of both CD105 and CD31 was observed into the tumor, indicating an effective specific targeting tumor vasculature mediated by TRC105. On the other hand, by analyzing other organs, like spleen and liver where graphene was detected by PET, there was no overlay of the two stained receptors, suggesting a main capture of the material by macrophages, rather than targeting of CD105. This is the first report of tumor targeting by graphene through antibody recognition. This work paves the way for the future use of graphene as a platform for drug delivery and photothermal therapy.

Recently, using a similar approach, Cornelissen et al. described the targeting and SPECT imaging of tumors using graphene oxide. ${ }^{46} \mathrm{GO}$ was covalently functionalized with Trastuzumab, an anti-HER2 antibody. Then, the metal ion chelator 2-(4-aminobenzyl)-diethylenetriaminepentaacetic acid (BnDTPA) was adsorbed by $\pi-\pi$ stacking onto the surface of GO and ${ }^{111}$ In was complexed to DTPA for radiolabeling studies (Fig. 4C). The effective targeting of the HER2-positive cell line by the functionalized GO was proved in vitro. Subsequent in vivo investigations were then conducted in both a xenografted and a spontaneous breast cancer model. SPECT imaging revealed a significant accumulation of GO into the tumor, probably due to a combined effect of the targeting properties of the antibody and the EPR effect, with a rapid clearance from the circulation.



Fig. 4 Graphene-based conjugates functionalized with antibodies for imaging. 


\section{Vaccination}

In the field of vaccination, the development of novel and efficient delivery strategies for the administration of protecting antigens is still challenging. In this context, carbon nanomaterials and in particular CNTs are promising because of their capacity to be internalized into a wide variety of cell types with minimal cytotoxicity. ${ }^{47}$ CNTs can therefore be exploited as carriers to administer vaccines by boosting the efficacy of antigens that cannot induce a sufficient and suitable response (Table 3). ${ }^{\mathbf{4 8}}$

In 2003, we first proposed the use of the CNTs for vaccination use by conjugating an antigen peptide. ${ }^{49}$ For this purpose, we functionalized CNTs with a peptide from the foot-and-mouth disease virus (FMDV), corresponding to the 141-159 region of the viral envelope protein VP1. This peptide was known to elicit neutralizing antibody responses that can afford complete protection of cattle against the disease. The peptide was covalently grafted to SWCNTs by a chemoselective approach (Fig. 5A). The nanotubes were first functionalized via 1,3-dipolar cycloaddition to introduce a triethylene glycol chain terminated by an ammonium group, which was subsequently derivatized with a maleimide linker. The $N$-terminal acetylated FMDV peptide, containing a cysteine at the $N$-terminal position, reacted with the maleimido-CNTs via a thiol-ene reaction. Surface plasmon resonance showed that the epitope structure of the peptide retained its antigenic form after conjugation to the CNTs. In addition, the enzyme-linked immunosorbent assay (ELISA) indicated that the peptide-CNT conjugate was recognized by polyclonal and monoclonal antibodies generated against the peptide. Both experiments suggested that the peptide covalently linked to the CNTs adopts the correct secondary conformation for antibody recognition. The peptideCNT conjugate resulted in immunogenic, eliciting virusneutralizing antibody responses in mice, while the CNTs nonfunctionalized with the peptide did not show intrinsic immunogenicity. ${ }^{50}$ This behavior was indicative of a successful presentation of the peptide linked to the CNTs in vivo. This work pointed out the potential of CNTs as carriers since no antibodies against the CNT structure were elicited. Thus, the antibodies were highly specific and the development of peptideCNT conjugates could find applications for vaccine delivery and diagnostic purposes.

In another study, we conjugated a Plasmodium vivax apical membrane antigen-1 (AMA-1) peptide to MWCNTs for the treatment of malaria (Fig. 5B). ${ }^{51}$ AMA-1 is a promising vaccine against Plasmodium, in the pre-erythrocytic and asexual blood stages, which brings about the mature form of the parasite. The peptide was covalently coupled to maleimido-functionalized MWCNTs via chemoselective ligation. The immunological characterization of the peptide-MWCNT conjugate indicated that the AMA-1 peptide was properly presented as the correct antigenic conformation was preserved and recognized by polyclonal antibodies. In vivo experiments using an infected Plasmodium berghei mouse model indicated that the conjugate had similar immunological properties compared to the peptide alone as it can protect or delay malarial infection. The immune response was peptide-specific and not directed towards CNTs, proving again that the nanotubes were not intrinsically immunogenic, resulting in an antigen-specific immune response. Based on our studies, the peptide presentation by functionalized CNTs to the immune system in vitro and in vivo is a promising approach for the development of new synthetic vaccine formulation based on carbon nanomaterials.

Scheinberg and coworkers have used CNTs as antigen carriers to improve vaccine therapy against other type of diseases and in particular cancer. ${ }^{52}$ The Wilm's tumor protein (WT1) is overexpressed in many human leukemias and other cancers and it is widely used in human trials as a cancer vaccine. Binding and presentation of clinically relevant cancerassociated peptide antigens by the major histocompatibility complex molecules of the antigen presenting cells allow

Table 3 Characteristics of CNT conjugates used for vaccination

\begin{tabular}{|c|c|c|c|c|c|}
\hline $\begin{array}{l}\text { Type of carbon } \\
\text { nanomaterial }\end{array}$ & Bioactive molecules & Functionalization & In vitro studies & In vivo studies & Reference \\
\hline SWCNTs & $\begin{array}{l}\text { Peptide from the foot- } \\
\text { and- } \\
\text { mouth disease virus }\end{array}$ & Covalent & BHK 21 cells & $\mathrm{BALB} / \mathrm{c}$ mice & 49 and 50 \\
\hline MWCNTs & $\begin{array}{l}\text { Plasmodium vivax apical } \\
\text { membrane antigen-1 } \\
\text { peptide }\end{array}$ & Covalent & - & $\begin{array}{l}\text { Infected Plasmodium } \\
\text { berghei mice }\end{array}$ & 51 \\
\hline MWCNTs & None & - & - & $\begin{array}{l}\text { MC38 murine colon } \\
\text { cancer xenografted } \\
\text { C57BL } 6 \text { mice }\end{array}$ & 53 \\
\hline
\end{tabular}






Fig. 5 Different types of CNTs (functionalized or non-functionalized) used for the development of new vaccination strategies for the treatment of different diseases.

inducing an effective immune response. But, these peptides are weakly immunogenic, a known characteristic of human tumor antigens. A solution to overcome this issue relies on the use of carbon nanomaterials to improve the immunogenicity of this type of peptides. In this study, SWCNTs were functionalized by 1,3-dipolar cycloaddition to generate amino groups on the sidewall (Fig. 5C) that were further derivatized to introduce benzaldehyde. The reaction between the aromatic aldehyde and a suitable $\mathrm{N}$-terminal hydrazine-modified peptide led to the formation of a covalent hydrazone bond, which is stable under physiologic conditions enough to perform cellular internalization and trafficking studies. For uptake studies, the peptide was also labeled with fluorescein moieties, whereas the SWCNTs were functionalized with a near-infrared fluorophore. The peptide and the nanotubes colocalized in peripheral vesicles and in a perinuclear compartment of the dendritic cells without affecting the cell viability. The conjugation of the peptide to SWCNTs maintained, but did not enhance the peptide-specific helper CD4 T cell response in vitro. However, mice immunized with the same conjugate and an adjuvant elicited enhanced specific IgG responses against the peptide. Control experiments demonstrated that the nanotube scaffold itself was nonimmunogenic, while the peptide alone did not induce an immune response in the presence of the adjuvant. This work demonstrates that CNTs can improve the immunogenicity of poorly immunogenic therapeutically relevant peptides.

The lack of appropriate activation of lymphocytes is one reason explaining the failure of the immune system to eradicate tumors. Embryonic stem cells (ESCs) can be used as a cancer vaccine to stimulate biological systems for destruction of colon cancer cells by eliciting an enhanced immune response. ESCs prevent and control the proliferation and expansion of malignant tumors in vivo via the de novo formation and maturation of $\mathrm{CD}^{+}$and $\mathrm{CD}^{+}{ }^{+} \mathrm{T}$ lymphocytes. With the objective to reach a stronger antitumor immunity by exploiting the role of ESCs in the proliferation of lymphocytes and the immunomodulating properties of CNTs, administration of non-functionalized MWCNTs and ESCs was performed in tumor-bearing mice (Fig. 5D). ${ }^{53}$ In this study, C57BL/6 mice inoculated with malignant colon cells were immunized with MWCNTs and ESCs, injected separately. The mice displayed remarkable suppression of both proliferation and development of malignant colon tumors in comparison with mice administered with only MWCNTs or ESCs. An enhanced proliferation of both $\mathrm{CD}^{+}{ }^{+}$and $\mathrm{CD}^{+}$lymphocytes, which are the main antitumor effector cells, was observed in the spleen of mice treated with the combination of MWCNTs and ESCs in comparison with the controls. The cytokine secretion also increased. The experiments suggest that the antitumor effect due to MWCNTs stems from the generation of IFN- $\gamma$ (interferon $\gamma$ ) and TNF (tumor necrosis factor) secreted by $\mathrm{CD}^{+}$cells. Overall, this work points out the potential of carbon nanotubes as anticancer immunization agents.

Other studies have reported the use of CNTs as scaffolds for the development of vaccines, in particular against tuberculosis. This disease is still considered as an important health concern worldwide. Tuberculosis can be prevented by vaccination, but this treatment is not always efficient. The disease causes about two million deaths per year; therefore there is a strong need to develop an improved vaccine. The current therapeutic strategy using the live attenuated Bacille Calmette-Guerin (BCG) vaccine is limited by potential risks as it is an attenuated wholeorganism viral vaccine. To overcome this limitation, a solution 
consists of specific macromolecules derived from the pathogen. In this context, the tuberculin purified protein derivative (PPD) from $M$. tuberculosis was conjugated to carboxylated SWCNTs (Fig. 5E) ${ }^{54}$ The cytotoxicity studies of the PPD-SWCNT conjugate showed no decrease in cell viability, neither nitric oxide production in splenocytes and peritoneal macrophages. The PPD-SWCNTs were then administered into BALB/c mice to evaluate the immune responses, in comparison with BCG, PPD alone, and PPD in a Freund's adjuvant emulsion (i.e. an antigen solution emulsified in mineral oil and used as an immunopotentiator). The immunization of mice with free PPD or PPD in Freund's adjuvant resulted in a T-helper type 2 (Th2) cytokine response (IL-5 and IL-10), whereas a mixed Th1 (IFN- $\gamma$ and IL12)/Th2 cytokine response was measured in the case of BCG. In contrast, the PPD-SWCNT conjugate induced a Th1-type cytokine response. As the protection against tuberculosis depends on the activation of Th1 immune response, this study shows that the vaccination with such conjugates could be an alternative strategy to BCG for immunization against $M$. tuberculosis in developing countries.

\section{Immunomodulation}

Few studies have also investigated the potential of CNTs to enhance and modulate immune responses. Indeed, an alternative approach for cancer immunotherapy relies on the stimulation of $\mathrm{T}$ cell immune responses against tumors via immunization with tumor antigens or by adoptive cell transfer therapy. T cells play a crucial role in initiating and maintaining immune responses (Table 4).

Oxidized SWCNTs have been used as platforms for physical adsorption of anti-CD3 antibodies, which are known to stimulate $\mathrm{T}$ cell proliferation, in order to enhance cellular activation (including both the magnitude and kinetics of $\mathrm{T}$ cell stimulation) (Fig. 6A). ${ }^{55}$ The clustering of these antibodies into the defect regions of SWCNTs created by the oxidative treatment led to an effective $\mathrm{T}$ cell stimulation. This effect can be explained by an increased avidity of the interaction to the antigen-presenting surface due to the formation of $\mathrm{T}$ cell antigen clusters, resulting in an enhanced recognition. It is known that antigen-presenting cells cluster antigenic ligands upon interfacing with $\mathrm{T}$ cells to increase the $\mathrm{T}$ cell response. In addition, the high specific surface area of SWCNTs allows adsorbing a high amount of proteins. The stimulation was more effective compared to similar concentrations of anti-CD3 alone. Moreover, the adsorption of the antibody onto other high surface area materials, such as activated carbon, polystyrene nanoparticles, and $\mathrm{C}_{60}$ fullerenes, did not result in equivalent levels of activation. Fluorescence resonance energy transfer microscopy showed the formation of large antibody stimulus clusters (5 to $6 \mu \mathrm{m}$ ) and a close inter-antibody distance (about $4.5 \mathrm{~nm}$ ) on the surface of oxidized SWCNTs. ${ }^{56}$ This work points out the potential of CNTs as a platform for artificial antigen presentation and efficient activation of lymphocytes. This therapeutic modality is certainly promising in immunotherapy, in particular for the development of artificial antigen-presentation platforms for ex vivo $\mathrm{T}$ cell stimulation and expansion in adoptive immunotherapy.

In the following study conducted by the same research group, the major histocompatibility complex class-I (MHC-I) loaded with a SIIN peptide was adsorbed on the sidewall of oxidized SWCNTs (Fig. 6A). ${ }^{57}$ The MHC-I complex was found to be denaturated when directly adsorbed on the nanotube surface. To preserve the active conformation, an alternative strategy was proposed by adsorbing neutravidin onto the oxidized SWCNTs, followed by non-covalent immobilization of the biotinylated MHC-I complex. In this case, the $\mathrm{T}$ cell response was enhanced more than three-fold compared to the soluble control under similar conditions. This result shows that protein adsorption can also be exploited to engineer CNTs for efficient presentation of MHC-I complexes for the antigenspecific stimulation of $\mathrm{T}$ cells.

CNTs have also been used as immunomodulators to boost the host immune activity in tumor-bearing mice. ${ }^{58}$ Subcutaneously administered oxidized MWCNTs induced significant activation of the complement system, promoted the production of inflammatory cytokines and activated the macrophages (Fig. 6B). These immunological responses inhibited the tumor growth progression. The oxidized MWCNTs were mainly taken

Table 4 Characteristics of CNT conjugates used for immunomodulation

\begin{tabular}{|c|c|c|c|c|c|}
\hline $\begin{array}{l}\text { Type of carbon } \\
\text { nanomaterial }\end{array}$ & Bioactive molecules & Functionalization & In vitro studies & In vivo studies & Reference \\
\hline SWCNTs & Anti-CD3 antibody & Non-covalent & $\mathrm{T}$ cells & & 55 and 56 \\
\hline MWCNTs & None & - & - & $\begin{array}{l}\text { Hepatocarcinoma } \\
\text { tumor-bearing mice }\end{array}$ & 58 \\
\hline MWCNTs & $\begin{array}{l}\text { Active agonist of antiviral } \\
\text { TLR7 receptor (7-TOG) }\end{array}$ & Covalent & $\begin{array}{l}\text { Human monocyte-derived } \\
\text { (Mo) DCs }\end{array}$ & - & 60 \\
\hline MWCNTs & None & - & Human myeloid DCs & - & 61 \\
\hline
\end{tabular}




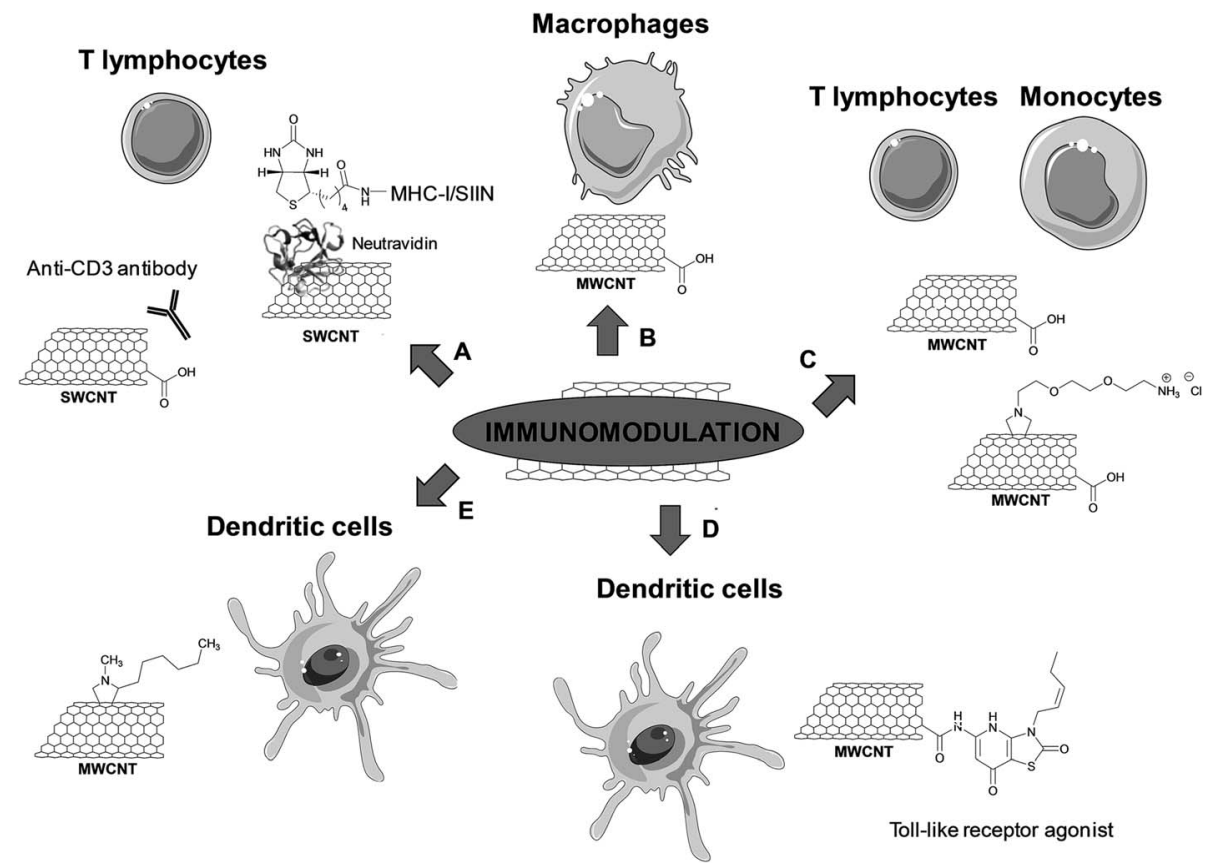

Fig. 6 Immunomodulation strategies using different types of functionalized CNTs.

up by macrophages that had been activated to perform phagocytosis. No sign of inflammation or accumulation of the CNTs in the liver, kidneys and spleen was observed. In addition, the nanotubes did not diffuse into the tumor cells. Thus, the tumor regression was not due to possible accumulation of CNTs into the tumor mass, but due to immunomodulation induced by the tubes. Some cytokines, which are relevant to enhance the immune functions of macrophages, $\mathrm{T}$ and $\mathrm{B}$ cells, were found to be upregulated. Therefore, this study gives attractive future prospects for the use of CNTs as a new class of adjuvant in antitumor immunotherapy.

Delogu and coworkers recently demonstrated the potential of CNTs as immunomodulators. ${ }^{59}$ Using the whole genome expression microarray technology, real-time polymerase chain reaction, and ELISA, transcriptomic changes induced by different types of functionalized CNTs in human immune cells (adaptive and innate) were evaluated. The types of MWCNTs used in this study differ in terms of diameter (i.e. 9.5 and 20$30 \mathrm{~nm}$ ) and functionalization (oxidized and amino-functionalized MWCNTs) (Fig. 6C). Three of the functionalized CNTs (i.e. 20-30 nm oxidized MWCNTs and both amino-functionalized MWCNTs) were able to activate immune-related pathways in monocytes, but not in $\mathrm{T}$ cells. These pathways are usually activated during acute inflammatory processes, for instance in the case of immune-mediated tumor rejection and clearance of pathogens. The fourth type of CNTs (i.e. $9.5 \mathrm{~nm}$ oxidized MWCNTs) was found to downregulate the genes associated with ribosomal proteins in both $\mathrm{T}$ cells and monocytes. These findings reveal that different CNTs can modulate the whole gene expression on immune cells. The CNTs activating the monocytes behave as cell specific immunostimulators, which opens new opportunities for use as immunotherapeutic agents and/or vaccine adjuvants.
The potential of CNTs as carriers has also been exploited to improve the immunogenic potential of DCs for cancer

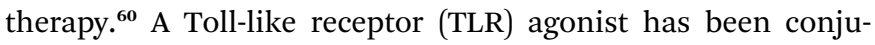
gated to CNTs for use as DC-based vaccines. For this purpose, oxidized MWCNTs have been covalently functionalized with 7-thia-8-oxoguanosine (7-TOG), which is an active agonist of the antiviral TLR7 receptor located within endosomal compartments (Fig. 6D). The activation of TLR7 leads to the maturation and migration of DCs, and to the improvement of the Th1-type immune response that could result in the eradication of tumor cells. The immunomodulatory effects of 7-TOG/CNTs on human DCs were investigated and their ability to stimulate human monocyte-derived (Mo) DCs was assessed. The 7-TOG/MWCNT conjugate was found to up-regulate the CD86 expression, allostimulatory activity, Th1- and Th17-polarizing capability of MoDCs. Such capability to activate MoDCs was not observed using the same concentration of 7-TOG or a mixture of oxidized MWCNTs and 7-TOG. Similar Th-polarizing capability was induced with only 10-times higher concentrations of 7-TOG. The higher efficacy of the covalent conjugate may stem from a higher intracellular concentration of 7-TOG thanks to the capacity of CNTs to penetrate into cells. Indeed, the 7-TOGMWCNTs were localized within the TLR $7^{+}$endocytic compartments of MoDCs, thus correlating well with higher expression of TLR7 in these compartments. This work demonstrates the potential use of CNTs as carriers of biostimulators of endosomal TLRs for the immunostimulation of DCs in cancer therapy.

The physico-chemical properties of CNTs can be exploited also in tissue engineering, in particular as scaffolds for bone regeneration and the formation of the cultured synaptic network. In a study by Ballerini and coworkers, it has been shown that solid tissue-growth platforms modify the adhesion 
properties of dendritic cells, which are professional antigenpresenting cells, and initiate different inflammatory responses. ${ }^{61}$ DCs are regulators of innate and adaptive immune responses or of tolerance induction. Artificial scaffolds can modify the adhesion of circulating DCs and tune immunogenic reactions. In this context, the interactions between functionalized MWCNT-based scaffolds and human myeloid DCs have been investigated. MWCNTs were functionalized by 1,3-dipolar cycloaddition with heptanal and sarcosine to introduce alkyl groups on the nanotube surface (Fig. 6E). A lower immunogenic profile of in vitro differentiated and activated DCs was observed when in contact with MWCNTs. Indeed, the DCs entangled to MWCNTs did not activate cell death programs. Interestingly, the DCs rather underwent a phenotypic and functional shift. This study demonstrates that a carbon material modified substrate is able to tune cell functions by intimate interactions with cells. Due to their morphology and physico-chemical properties, the CNTs can modulate the DC-mediated immune function.

\section{Conclusions}

Carbon nanomaterials have great potential in the biomedical domain thanks to their capacity to penetrate into cells. Within the different applications that have been envisaged, there are interesting prospects in immunotherapy. This therapeutic modality concerns the treatment of a disease by inducing, enhancing, or suppressing an immune response. In the case of antitumor vaccination, it is fundamental to activate the immune response, while for certain types of immune disease the responses need to be modulated or even suppressed. The possibility to chemically modify carbon nanomaterials allows creation of new systems that can be adapted to the specific immune intervention. Covalent and non-covalent functionalization of carbon nanotubes or graphene can be applied to conveniently attach targeting antibodies, imaging probes and/ or anticancer drugs. The combination of these three components can afford multifunctional systems that serve simultaneously for therapeutic and diagnostic purposes. We have here presented a series of interesting studies that have shown the potential of carbon nanomaterials in immunotherapy as they are able to increase the response to tumor antigens or to display immunomodulation effects. In addition, few studies have shown that carbon nanotubes are non-immunogenic, a key factor for the development of nanomaterials or nanoparticles as carriers for synthetic vaccines. Taking into consideration the fact that the toxicity of carbon nanomaterials can be also alleviated by using appropriate chemical approaches ${ }^{21}$ we believe that this field of research deserves future development and we would like to encourage further investigations for optimizing the conditions and allowing translation into future clinical studies.

\section{Acknowledgements}

This work was supported by the CNRS and partly by the ANR P2N (NANOTHER project 2010-NANO-008-398 04). CMM and AB acknowledge the EU FP7-ITN Marie-Curie Network programme RADDEL (290023). The authors wish to thank Hélène Dumortier for critically reading the manuscript.

\section{References}

1 L. Y. Rizzo, B. Theek, G. Storm, F. Kiessling and T. Lammers, Curr. Opin. Biotechnol., 2013, 24, 1159.

2 S. Chapman, M. Dobrovolskaia, K. Farahani, A. Goodwin, A. Joshi, H. Lee, T. Meade, M. Pomper, K. Ptak, J. Rao, R. Singh, S. Sridhar, S. Stern, A. Wang, J. B. Weaver, G. Woloschak and L. Yank, Nano Today, 2013, 8, 454.

3 S. Iijima, Nature, 1991, 354, 56.

4 M. F. De Volder, S. H. Tawfick, R. H. Baughman and A. J. Hart, Science, 2013, 339, 535.

5 D. Pantarotto, R. Singh, D. McCarthy, M. Erhardt, J.-P. Briand, M. Prato, K. Kostarelos and A. Bianco, Angew. Chem., Int. Ed., 2004, 43, 5242.

6 K. S. Novoselov, A. K. Geim, S. V. Morozov, D. Jiang, Y. Zhang, S. V. Dubonos, I. V. Grigorieva and A. A. Firsov, Science, 2004, 306, 666.

7 K. V. Krishna, C. Ménard-Moyon, S. Verma and A. Bianco, Nanomedicine, 2013, 8, 1669.

8 D. Peer, J. M. Karp, S. Hong, O. C. Farokhzad, R. Margalit and R. Langer, Nat. Nanotechnol., 2007, 2, 751.

9 Y. Zhang, Y. Bai and B. Yan, Drug Discovery Today, 2010, 15, 428.

10 N. Saito, Y. Usui, K. Aoki, N. Narita, M. Shimizu, K. Hara, N. Ogiwara, K. Nakamura, N. Ishigaki, H. Kato, S. Taruta and M. Endo, Chem. Soc. Rev., 2009, 38, 1897.

11 H. Y. Mao, S. Laurent, W. Chen, O. Akhavan, M. Imani, A. A. Ashkarran and M. Mahmoudi, Chem. Rev., 2013, 113, 3407.

12 K. Kostarelos, A. Bianco and M. Prato, Nat. Nanotechnol., 2009, 4, 627.

13 P. Singh, S. Campidelli, S. Giordani, D. Bonifazi, A. Bianco and M. Prato, Chem. Soc. Rev., 2009, 38, 2214.

14 D. A. Britz and A. N. Khlobystov, Chem. Soc. Rev., 2006, 35, 637.

15 V. Georgakilas, M. Otyepka, A. B. Bourlinos, V. Chandra, N. Kim, K. C. Kemp, P. Hobza, R. Zboril and K. S. Kim, Chem. Rev., 2012, 112, 6156.

16 G. Lamanna, A. Battigelli, C. Ménard-Moyon and A. Bianco, Nanotechnol. Rev., 2012, 1, 17.

17 V. Georgakilas, K. Kordatos, M. Prato, D. M. Guldi, M. Holzinger and A. Hirsch, J. Am. Chem. Soc., 2002, 124, 760.

18 Z. Liu, W. Cai, L. He, N. Nakayama, K. Chen, X. Sun, X. Chen and H. Dai, Nat. Nanotechnol., 2007, 2, 47.

19 N. W. S. Kam, Z. Liu and H. Dai, J. Am. Chem. Soc., 2005, 127, 12492.

20 C. Fabbro, H. Ali-Boucetta, T. Da Ros, K. Kostarelos, A. Bianco and M. Prato, Chem. Commun., 2012, 48, 3911.

21 H. Ali-Boucetta, A. Nunes, R. Sainz, M. A. Herrero, B. Tian, M. Prato, A. Bianco and K. Kostarelos, Angew. Chem., Int. Ed., 2013, 52, 2274. 
22 H. Dumortier, S. Lacotte, G. Pastorin, R. Marega, W. Wu, D. Bonifazi, J.-P. Briand, M. Prato, S. Muller and A. Bianco, Nano Lett., 2006, 6, 1522.

23 M. Orecchioni, D. Bedognetti, F. Sgarella, F. M. Marincola, A. Bianco and L. G. Delogu, J. Transl. Med., 2014, 12, 138.

24 K. T. Al-Jamal, A. Nunes, L. Methven, H. Ali-Boucetta, S. Li, F. M. Toma, M. A. Herrero, W. T. Al-Jamal, H. M. M. ten Eikelder, J. Foster, S. Mather, M. Prato, A. Bianco and K. Kostarelos, Angew. Chem., Int. Ed., 2012, 51, 6389.

25 N. Shao, S. Lu, E. Wickstrom and B. Panchapakesan, Nanotechnology, 2007, 18, 315101.

26 Y. Xiao, X. Gao, O. Taratula, S. Treado, A. Urbas, R. D. Holbrook, R. E. Cavicchi, C. T. Avedisian, S. Mitra, R. Savla, P. D. Wagner, S. Srivastava and H. He, $B M C$ Cancer, 2009, 9, 351.

27 P. Chakravarty, R. Marches, N. S. Zimmerman, A. D.-E. Swafford, P. Bajaj, I. H. Musselman, P. Pantano, R. K. Draper and E. S. Vitetta, Proc. Natl. Acad. Sci. U. S. A., 2008, 105, 8697.

28 R. Marches, P. Chakravarty, I. H. Musselman, P. Bajaj, R. N. Azad, P. Pantano, R. K. Draper and E. S. Vitetta, Int. J. Cancer, 2009, 125, 2970.

29 J. Meng, J. Meng, J. Duan, H. Kong, L. Li, C. Wang, S. Xie, S. Chen, N. Gu, H. Xu and X.-D. Yang, Small, 2008, 4, 1364.

30 A. Bianco, J. Hoebeke, S. Godefroy, O. Chaloin, D. Pantarotto, J.-P. Briand, S. Muller, M. Prato and C. D. Partidos, J. Am. Chem. Soc., 2005, 127, 58.

31 D. Zhao, D. Alizadeh, L. Zhang, W. Liu, O. Farrukh, E. Manuel, D. J. Diamond and B. Badie, Clin. Cancer Res., 2011, 17, 771.

32 A. F. Carpentier, G. Auf and J. Y. Delattre, Front. Biosci., 2003, 8, e115.

33 H. Fan, I. Zhang, X. Chen, L. Zhang, H. Wang, A. Da Fonseca, E. R. Manuel, D. J. Diamond, A. Raubitschek and B. Badie, Clin. Cancer Res., 2012, 18, 5628.

34 J. Sun, J. Chao, J. Huang, M. Yin, H. Zhang, C. Peng, Z. Zhong and N. Chen, ACS Appl. Mater. Interfaces, 2014, 6, 7926.

35 R. Yang, X. Yang, Z. Zhang, Y. Zhang, S. Wang, Z. Cai, Y. Jia, Y. Ma, C. Zheng, Y. Lu, R. Roden and Y. Chen, Gene Ther., 2006, 13, 1714.

36 L. Shen, K. Evel-Kabler, R. Strube and S. Y. Chen, Nat. Biotechnol., 2004, 22, 1546.

37 R. Li, R. Wu, L. Zhao, M. Wu, L. Yang and H. Zou, ACS Nano, 2010, 4, 1399.

38 C. Sacchetti, N. Rapini, A. Magrini, E. Cirelli, S. Bellucci, M. Mattei, N. Rosato, N. Bottini and M. Bottini, Bioconjugate Chem., 2013, 24, 852.

39 M. R. McDevitt, D. Chattopadhyay, B. J. Kapper, J. S. Jaggi, S. R. Schiffman, C. Antczak, J. T. Njardarson, R. Brentjens and D. A. Scheinberg, J. Nucl. Med., 2007, 48, 1180.

40 A. Ruggiero, C. H. Villa, J. P. Holland, S. R. Sprinkle, C. May, J. S. Lewis, D. A. Scheinberg and M. R. McDevitt, Int. J. Nanomed., 2010, 5, 783.

41 J. J. Mulvey, C. H. Villa, M. R. McDevitt, F. E. Escorcia, E. Casey and D. A. Scheinberg, Nat. Nanotechnol., 2013, 8, 763.
42 C. Staii, A. T. Johnson, M. Chen and A. Gelperin, Nano Lett., 2005, 5, 1774.

43 S. Shi, K. Yang, H. Hong, H. F. Valdovinos, T. R. Nayak, Y. Zhang, C. P. Theuer, T. E. Barnhart, Z. Liu and W. Cai, Biomaterials, 2013, 34, 3002.

44 H. Hong, K. Yang, Y. Zhang, J. W. Engle, L. Feng, Y. Yang, T. R. Nayak, S. Goel, J. Bean, C. P. Theuer, T. E. Barnhart, Z. Liu and W. Cai, ACS Nano, 2012, 6, 2361.

45 H. Hong, Y. Zhang, J. W. Engle, T. R. Nayak, C. P. Theuer, R. J. Nickles, T. E. Barnhart and W. Cai, Biomaterials, 2012, 33, 4147.

46 B. Cornelissen, S. Able, V. Kersemans, P. A. Waghorn, S. Myhra, K. Jurkshat, A. Crossley and K. A. Vallis, Biomaterials, 2013, 34, 1146.

47 K. Kostarelos, L. Lacerda, G. Pastorin, W. Wu, S. Wieckowski, J. Luangsivilay, S. Godefroy, D. Pantarotto, J. P. Briand, S. Muller, M. Prato and A. Bianco, Nat. Nanotechnol., 2007, 2, 108 .

48 D. A. Scheinberg, M. R. McDevitt, T. Dao, J. J. Mulvey, E. Feinberg and S. Alidori, Adv. Drug Delivery Rev., 2013, 65, 2016.

49 D. Pantarotto, C. D. Partidos, R. Graff, J. Hoebeke, J. P. Briand, M. Prato and A. Bianco, J. Am. Chem. Soc., 2003, 125, 6160.

50 D. Pantarotto, C. D. Partidos, J. Hoebeke, F. Brown, E. Kramer, J. P. Briand, S. Muller, M. Prato and A. Bianco, Chem. Biol., 2003, 10, 961.

51 N. Yandar, G. Pastorin, M. Prato, A. Bianco, M. E. Patarroyo and J. M. Lozano, Vaccine, 2008, 26, 5864.

52 C. H. Villa, T. Dao, I. Ahearn, N. Fehrenbacher, E. Casey, D. A. Rey, T. Korontsvit, V. Zakhaleva, C. A. Batt, M. R. Philips and D. A. Scheinberg, ACS Nano, 2011, 5, 5300. 53 T. Mocan and C. Iancu, Int. J. Nanomed., 2011, 6, 1945.

54 M. Zeinali, M. Jammalan, S. K. Ardestani and N. Mosaveri, Immunol. Lett., 2009, 126, 48.

55 T. R. Fadel, E. R. Steenblock, E. Stern, N. Li, X. Wang, G. L. Haller, L. D. Pfefferle and T. M. Fahmy, Nano Lett., 2008, 8, 2070.

56 T. R. Fadel, M. Look, P. A. Staffier, G. L. Haller, L. D. Pfefferle and T. M. Fahmy, Langmuir, 2010, 26, 5645.

57 T. R. Fadel, N. Li, S. Shah, M. Look, L. D. Pfefferle, G. L. Haller, S. Justesen, C. J. Wilson and T. M. Fahmy, Small, 2013, 9, 666.

58 J. Meng, M. Yang, F. Jia, H. Kong, W. Zhang, C. Wang, J. Xing, S. Xie and H. Xu, Nanotechnology, 2010, 21, 145104.

59 M. Pescatori, D. Bedognetti, E. Venturelli, C. Ménard-Moyon, C. Bernardini, E. Muresu, A. Piana, G. Maida, R. Manetti, F. Sgarrella, A. Bianco and L. G. Delogu, Biomaterials, 2013, 34, 4395.

60 M. Čolić, T. Džopalić, S. Tomić, J. Rajković, R. Rudolf, G. Vuković, A. Marinković and P. Uskoković, Carbon, 2014, 67, 273.

61 A. Aldinucci, A. Turco, T. Biagioli, F. M. Toma, D. Bani, D. Guasti, C. Manuelli, L. Rizzetto, D. Cavalieri, L. Massacesi, T. Mello, D. Scaini, A. Bianco, L. Ballerini, M. Prato and C. Ballerini, Nano Lett., 2013, 13, 6098. 